\title{
Decision Directed Channel Estimation Employing Projection Approximation Subspace Tracking
}

\author{
Jos Akhtman and Lajos Hanzo \\ School of ECS., Univ. of Southampton, SO17 1BJ, UK. \\ Tel: +44-23-80-593 125, Fax: +44-23-80-593 045 \\ Email: lh@ecs.soton.ac.uk, http://www-mobile.ecs.soton.ac.uk
}

\begin{abstract}
The attainable capacity and integrity of a state-of-the-art broadband multi-carrier communication system is highly sensitive to the accuracy of the information available concerning the channel conditions encountered. The majority of existing advanced channel estimation schemes assume knowledge of the channel's Power Delay Profile (PDP) in order to estimate the corresponding instanteneous Channel Impulse Response (CIR). It is evident however that in realistic mobile channels, where at least one of the communicating terminals is in motion, the power delay profile will also be time-variant and thus may not be known a priori at the receiver. In this paper we propose a decision directed channel estimation scheme employing the so-called Projection Approximation Subspace Tracking (PAST) algorithm. The PAST algorithm is utilised for the sake of achieving low-complexity recursive tracking of the channel's PDP. The achievable perormance of the proposed method is documented in the context of an OFDM system communicating in realistic channel conditions characterized by a time-variant fractionally-spaced PDP.
\end{abstract}

\section{INTRODUCTION}

The ever-increasing demand for high data-rates in wireless networks requires the efficient utilisation of the limited bandwidth available, while supporting a high grade of mobility in diverse propagation environments. On the other hand, Orthogonal Frequency Devision Multiplexing (OFDM) [1] is capable of satisfying these requirements. This is a benefit of its ability to cope with highly time-variant wireless channel characteristics. However, as pointed out in [2], the capacity and the achievable integrity of communication systems is highly dependent on the system's knowledge concerning the channel conditions encountered. Thus, the provision of an accurate and robust channel estimation strategy is a crucial factor in achieving a high performance.

The family of well-documented decision directed channel estimation (DDCE) methods [1], [3]-[5] provides a suitable solution for the problem of channel estimation in OFDM-based systems. The major benefit of the DDCE scheme is that in contrast to purely pilot assisted channel estimation methods [6], [7] both the pilot symbols as well as all the information symbols are utilised for channel estimation [1]. The simple philosophy of this method is that in the absence of transmission errors we can benefit from the availability of $100 \%$ pilot information by using the detected subcarrier symbols as an $a$ posteriori reference signal. The employment of this method allows us to reduce the number of pilot symbols required.

The majority of the aforementioned channel estimation methods rely on the a priori knowlege of the channel statistics commonly characterized by the channel's Power Delay Profile (PDP) for the sake of estimating the instantenious Channel Impulse Response and the corresponding frequency-domain Channel Transfer Function (CTF). It is evident however that in realistic wireless mobile channels,

Acknowledgements: The work reported in this paper has formed part of the Wireless Enabling Techniques work area of the Core 4 Research Programme of the Virtual Centre of Excellence in Mobile and Personal Communications, Mobile VCE, www.mobilevce.com, whose funding support, including that of EPSRC, is gratefully acknowledged. Fully detailed technical reports on this research are available to Industrial Members of Mobile VCE. where at least one of the communicating terminals is in motion, the channel's PDP will also become time-variant and thus may not be a priori known at the receiver. In this paper we propose a decisiondirected channel estimation method, which employs the so-called Projection Approximation Subspace Tracking (PAST) algorithm [8] for the sake of recursive tracking of the channel's PDP and subsequent estimation of the instantaneous CTF.

\section{SySTEM MODEL}

The discrete frequency-domain model of the OFDM system can be described as in [9]

$$
y[n, k]=H[n, k] x[n, k]+w[n, k],
$$

for $k=0, \ldots, K-1$ and all $n$, where $y[n, k], x[n, k]$ and $w[n, k]$ are the received symbol, the transmitted symbol and the Gaussian noise sample respectively, corresponding to the $k$ th subcarrier of the $n$th OFDM block. Furthermore, $H[n, k]$ represents the complexvalued Channel Transfer Function (CTF) coefficient associated with the $k$ th subcarrier and time instance $n$. In the case of an $M$ QAM modulated OFDM system, $x[n, k]$ corresponds to the M-QAM symbol accommodated by the $k$ th subcarrier.

As it was pointed out in [5], in OFDM systems using a sufficiently long cyclic prefix and adequate synchronisation, the subcarrier-related CTF can be expressed as

$$
H[n, k] \triangleq H(n T, k \Delta f)=C(k \Delta f) \sum_{l=1}^{L} \alpha_{l}[n] W_{K}^{k \tau_{l} / T_{s}},
$$

where $\alpha[n]$ is the fractionally-spaced CIR and $C(f)$ is an aggregate transfer function of the transmitter-receiver pulse-shaping filter pair. Note that in realistic channel conditions associated with non-samplespaced time-variant path-delays $\tau_{l}(n)$ the fractionally-spaced CIR (FS-CIR) $\alpha_{l}[n] \triangleq \alpha_{l}(n T)$ will be constituted by a low number of $L$ non-zero statistically independent taps associated with distinctive propagation paths. The corresponding PDP $\lambda_{l}[n]$ constituted by the Mean Square (MS) values $\mathrm{E}\left\{\left|\alpha_{l}[n]\right|^{2}\right\}$ may be identified as the vector of the eigenvalues of the channel's covariance matrix

$$
\boldsymbol{C}_{H}=\mathrm{E}\left\{\boldsymbol{H}[n] \boldsymbol{H}^{\mathrm{H}}[n]\right\}=\boldsymbol{W}[n] \operatorname{diag}\left(\lambda_{l}[n]\right) \boldsymbol{W}^{\mathrm{H}}[n],
$$

where $\boldsymbol{W}$ is a $(K \times L)$-dimensional matrix having the eigenvectors of the channel covariance matrix $C_{H}[n]$ as its columns. The columns of the transformation matrix $W$ span the so-called signal subspace of the random vector process associated with the subcarrier-related CTF $\boldsymbol{H}[n]$. Furthermore, Equation (2) can be expressed in a matrix form as

$$
\boldsymbol{H}[n]=\boldsymbol{W}[n] \boldsymbol{\alpha}[n] .
$$

\section{PARAMETRIC FS-CIR Estimation}

\section{A. Projection Approximation Subspace Tracking}

Let $\boldsymbol{H}[n] \in \mathbb{C}^{K}$ be the vector of the subcarrier-related CTF coefficients associated with the channel model of Equation (1). As 
described in Section II, the CIR associated with the CTF coefficient vector $\boldsymbol{H}[n]$ is constituted by a relatively low number of $L \ll K$ statistically-independent Rayleigh fading paths. The corresponding CIR components are related to the CTF coefficients $H[n, k]$ by means of Equation (2). The motivation for employing the so-called subspace technique [10] here is that usually we have $L \ll K$ and thus it is more efficient to estimate a low number of CIR-related taps in the low-dimensional signal subspace than estimating all the $K$ FD-CTF coefficients.

Let $\lambda_{l}$ and $\boldsymbol{u}_{l}$ be the eigenvalues and the corresponding eigenvectors of the CTF's covariance matrix $C_{H}$ of Equation (3). Then, we have $\boldsymbol{C}_{H}=\boldsymbol{U} \boldsymbol{\Sigma} \boldsymbol{U}^{\mathrm{H}}$, where $\boldsymbol{\Sigma}=\operatorname{diag}\left(\lambda_{l}\right)$ and $\boldsymbol{U}=\left[\boldsymbol{u}_{1} \cdots \boldsymbol{u}_{K}\right]$.

The eigenvalues aligned in a descending order may be expressed as

$$
\lambda_{1} \geq \cdots \geq \lambda_{L}>\lambda_{L+1}=\cdots=\lambda_{K}=\sigma_{w}^{2}
$$

where the first $L$ dominant eigenvalues $\lambda_{1}, \cdots, \lambda_{L}$ in conjunction with the $L$ corresponding eigenvectors $\boldsymbol{u}_{1}, \cdots, \boldsymbol{u}_{L}$ may be termed as the signal eigenvalues and eigenvectors, respectively [8]. The remaining eigenvalues $\lambda_{L+1}, \cdots, \lambda_{K}$ and eigenvectors $\boldsymbol{u}_{L+1}, \cdots, \boldsymbol{u}_{K}$ are termed the noise eigenvalues and eigenvectors. The resultant sets of signal and noise eigenvectors, which are column vectors, span the mutually orthogonal signal and noise subspaces $U_{S}$ and $U_{N}$, such that we have

$$
\boldsymbol{U}_{S}=\left[\boldsymbol{u}_{1}, \cdots, \boldsymbol{u}_{L}\right] \quad \text { and } \quad \boldsymbol{U}_{N}=\left[\boldsymbol{u}_{L+1}, \cdots, \boldsymbol{u}_{K}\right] .
$$

The corresponding time-domain-related $L$-tap estimate of the FS-CIR vector $\alpha[n]$ may be obtained as follows

$$
\hat{\boldsymbol{\alpha}}=\boldsymbol{U}_{S}^{\mathrm{H}}[n] \tilde{\boldsymbol{H}}[n] .
$$

Furthermore, the reduced-noise estimate of the CTF vector $\boldsymbol{H}[n]$ may reconstructed using

$$
\hat{\boldsymbol{H}}[n]=\boldsymbol{U}_{S}[n] \hat{\boldsymbol{\alpha}}[n] .
$$

For the sake of evaluating and tracking the potentially time-variant signal subspace $U_{S}[n]$ we employ subspace tracking method developed by Yang [8]. More specifically, we consider the following real-valued scalar objective function having the matrix argument of $W \in \mathbb{C}^{K \times L}$

$$
\begin{aligned}
J(\boldsymbol{W}) & =\mathrm{E}\left\{\left\|\boldsymbol{H}-\boldsymbol{W} \boldsymbol{W}^{\mathrm{H}} \boldsymbol{H}\right\|^{2}\right\} \\
& =\operatorname{tr}\left(\boldsymbol{C}_{H}\right)-2 \operatorname{tr}\left(\boldsymbol{W}^{\mathrm{H}} \boldsymbol{C}_{H} \boldsymbol{W}\right)+\operatorname{tr}\left(\boldsymbol{W}^{\mathrm{H}} C_{H} \boldsymbol{W} \cdot \boldsymbol{W}^{\mathrm{H}} \boldsymbol{W}\right)
\end{aligned}
$$

As demonstrated by Yang in [8], the objective function $J(\boldsymbol{W})$ of Equation (9) exhibits the following important properties

1) $W$ is a stationary point of $J(W)$ if and only if we have $\boldsymbol{W}=\boldsymbol{U}_{L} \boldsymbol{Q}$, where $\boldsymbol{U}_{L} \in \mathbb{C}^{K \times L}$ contains any $L$ distinct eigenvectors of $C_{H}$ and $Q \in \mathbb{C}^{L \times L}$ is an arbitrary unitary matrix. Furthermore, at each stationary point, $J(\boldsymbol{W})$ equals the sum of these particular eigenvalues, whose eigenvectors are not involved in $\boldsymbol{U}_{L}$ [8, Theorem 1].

2) All stationary points of $J(\boldsymbol{W})$ are local saddle points except, when $U_{L}$ contains the $L$ dominant eigenvectors of $C_{H}$. In this case, $J(\boldsymbol{W})$ attains the global minimum [8, Theorem 2].

3) The global convergence of $W$ is guaranteed by using iterative minimization of $J(\boldsymbol{W})$ and the columns of the resultant value of $\boldsymbol{W}$ will span the signal subspace of $C_{H}$.

4) The use of an iterative algorithm to minimize $J(\boldsymbol{W})$ will always converge to an orthonormal basis of the signal subspace of $C_{H}$ without invoking any orthonormalization operations during the iterations.
5) The global minimum of $J(\boldsymbol{W}), \boldsymbol{W}$ does not necessarily contain the signal eigenvectors, but an arbitrary orthogonal basis of the signal subspace of $C_{H}$ as indicated by the unitary matrix $Q$ introduced in Property 1 . In other words, we have $W=$ $\operatorname{argmin} J(\boldsymbol{W})$ if and only if $\boldsymbol{W}=\boldsymbol{U}_{S} \boldsymbol{Q}$, where $\boldsymbol{Q}$ is an arbitrary unitary matrix.

6) For the simple scalar case of $L=1$, the solution minimizing $J(\boldsymbol{W})$ is given by the most dominant normalized eigenvector of $C_{H}$.

Subsequently, Yang [8] proposes an iterative RLS algorithm for tracking of the signal subspace of the channel's covariance matrix $C_{H}$. Specifically, upon replacing the expectation value in Equation (9) by the exponentially weighted sum of the RLS algorithm, we arrive at the following new objective function

$$
J(\boldsymbol{W}[n])=\sum_{i=1}^{n} \eta^{n-i}\left\|\boldsymbol{H}[i]-\boldsymbol{W}[n] \boldsymbol{W}^{\mathrm{H}}[n] \boldsymbol{H}[i]\right\|^{2},
$$

where $\eta \in(0,1)$ is the so-called forgetting factor, which accounts for possible deviations of the actual channel statistics encountered from the WSS assumption. Observe that the sole difference between the objective functions of Equations (9) and (10) is the introduction of the time-variant exponentially weighted sample covariance matrix [8], which may be expressed as

$$
\boldsymbol{C}_{H}[n]=\sum_{m=1}^{n} \eta^{n-m} \boldsymbol{H}[m] \boldsymbol{H}^{\mathrm{H}}[m]=\eta \boldsymbol{C}_{H}[n-1]+\boldsymbol{H}[n] \boldsymbol{H}^{\mathrm{H}}[n]
$$

instead of the time-invariant matrix $C_{H}=\mathrm{E}\left\{\boldsymbol{H} \boldsymbol{H}^{\mathrm{H}}\right\}$ of Equation (3).

The PAST algorithm may be derived by approximating the expression $\boldsymbol{W}^{\mathrm{H}}[n] \boldsymbol{H}[m]$ in Equation (10), which may be interpreted as a projection of the vector $\boldsymbol{H}[m]$ onto the column space of the matrix $\boldsymbol{W}[n]$, by the readily available a posteriori vector $\boldsymbol{\alpha}[m]=$ $\boldsymbol{W}^{\mathrm{H}}[m] \boldsymbol{H}[m]$. The resultant modified cost function may be formulated as

$$
J^{\prime}(\boldsymbol{W}[n])=\sum_{m=1}^{n} \eta^{n-m}\|\boldsymbol{H}[m]-\boldsymbol{W}[n] \boldsymbol{\alpha}[m]\|^{2} .
$$

As is argued in [8], for stationary or slowly varying signals, the aforementioned projection approximation, hence the name PAST, does not substantially change the error surface associated with the corresponding cost function of Equation (12) and therefore does not significantly affect the convergence properties of the derived algorithm.

Similarly to other RLS estimation schemes [11], [12], the cost function $J^{\prime}(\boldsymbol{W}[n])$ is minimized if

$$
\boldsymbol{W}=\boldsymbol{C}_{H \alpha}[n] \boldsymbol{C}_{\alpha \alpha}^{-1}[n],
$$

where we have

$$
\boldsymbol{C}_{H \alpha}[n]=\sum_{i=1}^{n} \eta^{n-i} \boldsymbol{H}[i] \boldsymbol{\alpha}^{\mathrm{H}}[i]=\eta \boldsymbol{C}_{H \alpha}[n-1]+\boldsymbol{H}[n] \boldsymbol{\alpha}^{\mathrm{H}}[n]
$$

and

$$
C_{\alpha \alpha}[n]=\sum_{i=1}^{n} \eta^{n-i} \boldsymbol{\alpha}[i] \boldsymbol{\alpha}^{\mathrm{H}}[i]=\eta \boldsymbol{C}_{\alpha \alpha}[n-1]+\boldsymbol{\alpha}[n] \boldsymbol{\alpha}^{\mathrm{H}}[n] .
$$

Following the RLS approach [8], a low-complexity solution of the computational problem associated with minimizing the cost function $J^{\prime}(\boldsymbol{W}[n])$ of Equation (12) may be obtained using recursive updates of the matrix $\boldsymbol{W}[n]$. More specifically, we have

$$
\boldsymbol{W}[n]=\boldsymbol{W}[n-1]+\boldsymbol{e}[n] \boldsymbol{k}^{\mathrm{H}}[n],
$$


Algorithm 1 Deflation PAST

$$
\begin{aligned}
& \boldsymbol{H}_{1}[n]=\boldsymbol{H}[n] \\
& \text { for } \quad l=1,2, \ldots, L \text { do } \\
& \quad \alpha_{l}[n]=\boldsymbol{w}_{l}^{\mathrm{H}}[n-1] \boldsymbol{H}_{l}[n] \\
& \quad \lambda_{l}[n]=\beta \lambda_{l}[n-1]+\left|\alpha_{l}[n]\right|^{2} \\
& \quad \boldsymbol{e}_{l}[n]=\boldsymbol{H}_{l}[n]-\boldsymbol{w}_{l}[n-1] \alpha_{l}[n] \\
& \quad \boldsymbol{w}_{l}[n]=\boldsymbol{w}_{l}[n-1]+\boldsymbol{e}_{l}[n]\left(\alpha_{l}^{*}[n] / \lambda_{l}[n]\right) \\
& \quad \boldsymbol{H}_{l+1}[n]=\boldsymbol{H}_{l}[n]-\boldsymbol{w}_{l}[n] \alpha_{l}[n] \\
& \text { end for }
\end{aligned}
$$

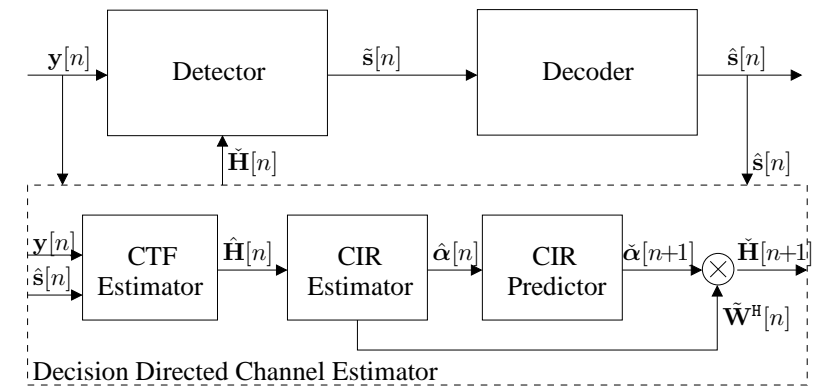

Fig. 1. Schematics of a generic receiver employing Decision Directed Channel Estimator constituted by an a posteriori decision-directed CTF Estimator, followed by a CIR Estimator and an a priori CIR predictor.

\section{PASTD -Aided FS-CIR Estimation}

obtained as

$$
\boldsymbol{e}[n]=\boldsymbol{H}[n]-\boldsymbol{W}[n-1] \boldsymbol{\alpha}[n-1],
$$

while

$$
\boldsymbol{k}[n]=\frac{\boldsymbol{P}[n-1] \boldsymbol{\alpha}[n]}{\eta+\boldsymbol{\alpha}^{\mathrm{H}}[n] \boldsymbol{P}[n-1] \boldsymbol{\alpha}[n]}
$$

denotes the RLS gain vector. Furthermore, the matrix $\boldsymbol{P}[n]$ is the inverse of the CIR-related taps' $(L \times L)$-dimensional covariance matrix $C_{\alpha \alpha}$, which can be recursively calculated as follows

$$
\boldsymbol{P}[n]=\frac{1}{\eta} \operatorname{Tri}\left\{\left(\boldsymbol{I}-\boldsymbol{k}[n] \boldsymbol{\alpha}^{\mathrm{H}}[n]\right) \boldsymbol{P}[n-1]\right\},
$$

where the operator $\operatorname{Tri}\{\cdot\}$ indicates that only the upper triangular part of $P[n]$ is calculated and its Hermitian conjugate version is copied to the lower triangular part [8].

\section{B. Deflation PAST}

In this work, however, we aim for maintaining the lowest possible complexity hence we are particularly interested in the deflation-based version of the PAST algorithm derived in [8], which is referred to as the PASTD algorithm. The simple philosophy of the deflation method is the sequential estimation of the principal components of the CTF covariance matrix $C_{H}$ [13]. Consequently, we first update the most dominant eigenvector $w_{1}[n]$ by applying the PAST method of Section III-A in conjunction with $L=1$. Subsequently, the projection of the current sample vector $\boldsymbol{H}[n]$ onto the updated eigenvector $w_{1}[n]$ is subtracted from itself, resulting in a modified (deflated) version of the CTF vector in the following form $\boldsymbol{H}_{2}[n]=$ $\boldsymbol{H}[n]-\boldsymbol{w}_{1}[n] \boldsymbol{w}_{1}^{\mathrm{H}}[n] \boldsymbol{H}[n]$. The second most dominant eigenvector $w_{2}[n]$ has now become the most dominant one and therefore may be updated similarly to $w_{1}[n]$. By repeatedly applying this procedure, all the desired eigencomponents may be estimated. The resultant PASTD method is summarized in Algorithm 1.

A particularly important property of the PASTD method of Algorithm 1 is that as opposed to the PAST method of Section III-A, it enables the explicit tracking of the time-variant eigencomponents of the channel covariance matrix $C_{H}[n]$, namely the eigenvectors $w_{l}[n]$ as well as of the corresponding eigenvalues $\lambda_{l}[n]$ according to

$$
\boldsymbol{w}_{l}[n]=\boldsymbol{w}_{l}[n-1]+\frac{\alpha_{l}^{*}[n]}{\lambda_{l}[n]}\left(\boldsymbol{H}_{l}[n]-\boldsymbol{w}_{l}[n-1] \alpha_{l}[n]\right)
$$

where we have $\boldsymbol{\alpha}_{l}[n]=\boldsymbol{w}_{l}^{\mathrm{H}}[n-1] \boldsymbol{H}[n]$ and $\lambda_{l}[n]=\beta \lambda_{l}[n-1]+$ $\left|\alpha_{l}[n]\right|^{2}$.
In this section we would like to utilize the PASTD method detailed in Section III-B in the context of the decision-directed channel estimation scheme depicted in Figure 1.

More specifically, our channel estimator is constituted by what we refer to as an a posteriori decision-directed CTF estimator followed by an a posteriori FS-CIR estimator and an a priori CIR predictor [1]. We consider a PASTD -aided a posteriori FS-CIR estimator, which corresponds to the CIR Estimator module of Figure 1. As seen in Figure 1, the task of the CIR estimator is to estimate the FS-CIR taps of Equation (2).

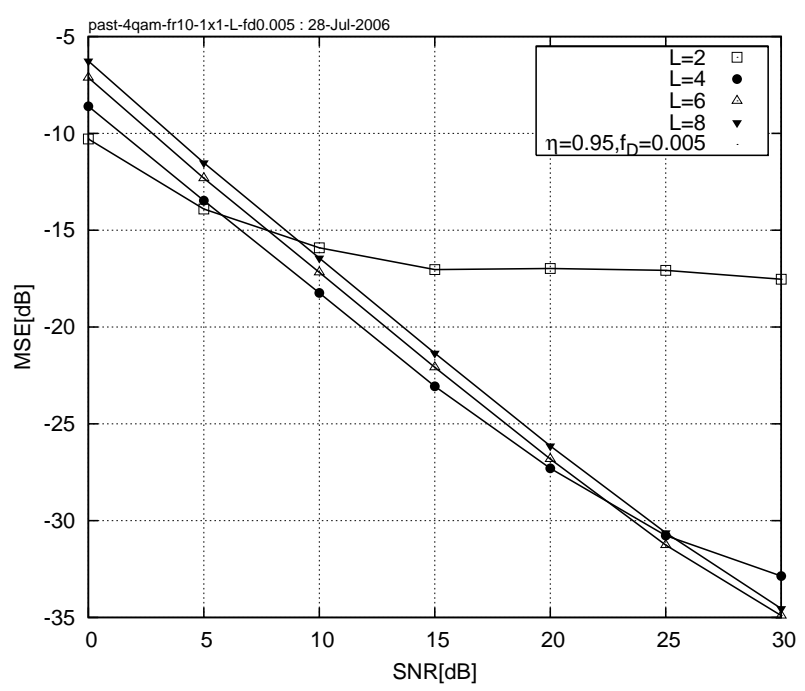

Fig. 2. MSE versus SNR performance exhibited by the 4QAM-OFDM system employing the PASTD CIR estimator of Algorithm 1 and tracking $L=2,4,6$ and 8 CIR taps. The value of the PASTD forgetting factor was $\eta=0.95$. The Doppler frequency was $f_{D}=0.005$. Additional system parameters are summarized in Table I.

The achievable performance of the subspace tracking method of Section III-B is characterized in Figures 2, 3 and 4, where we define the Mean Square Error (MSE) performance criterion as follows

$$
M S E=\mathrm{E}\left\{\sum_{l}\left|e_{l}[n]\right|^{2}\right\},
$$

where $e_{l}$ is the FD-CTF tracking error defined by Equation 17. In our simulations we consider an OFDM system having $K=128$ orthogonal QPSK-modulated subcarriers. The system characteristics are outlined in Table I. We employ an OFDM-frame-variant channel model having a time-variant 8-tap PDP characterized by the COST207 BU channel model [14]. Additionally, each individual propa- 


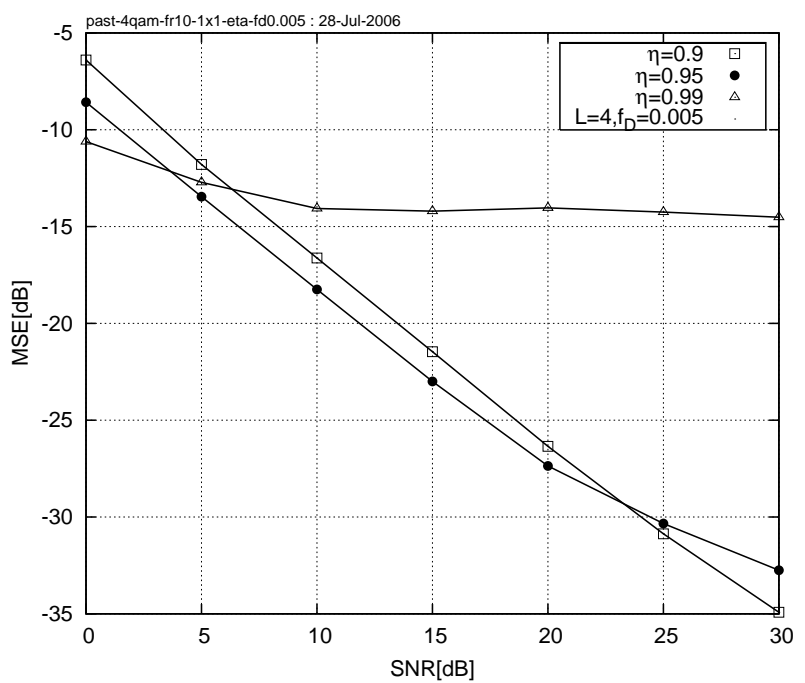

Fig. 3. MSE versus SNR performance exhibited by the 4QAM-OFDM system employing the PASTD method of Algorithm 1. The values of the PASTD forgetting factor wwere $\eta=0.9,0.95$ and 0.9. The Doppler frequency was $f_{D}=0.005$. Additional system parameters are summarized in Table I.

gation path undergoes fast Rayleigh fading with a corresponding OFDM-symbol-normalized Doppler frequency of $f_{D}=0.005$. The resultant channel can be characterised as a mutli-path Rayleighfading channel with slowly-varying PDP, where the relative delays $\tau_{l}$ associated with different PDP taps vary with time at a rate determined by the drift rate parameter $v_{\tau}$

Firstly, Figure 2 characterizes the achievable FD-CTF MSE performance of the PASTD method of Algorithm 1 for different ranks $L$ of the estimated subspace, while assuming a constant value of $\eta=0.95$ for the forgetting factor. From Figure 2, we may conclude that a high CIR estimator performance may be achieved when assuming that the estimated CTF signal subspace has a rank of $L=4$, regardless of the actual number of paths constituting the multi-path channel encountered.

Secondly, Figure 3 characterizes the achievable MSE performance of the PASTD method of Algorithm 1 for different values of the forgetting factor $\eta$, while assuming a constant rank of $L=4$ for the estimated subspace. As may be concluded from Figure 3, the optimum value of the forgetting factor $\eta$ is largely dependent on the SNR as well as on the Doppler frequency encountered. Nevertheless, the compromise value of $\eta=0.95$ appears to constitute a relatively good choice in the practical ranges of both SNR values and Doppler frequencies.

Finally, Figure 4 characterizes the achievable MSE performance of the PASTD method of Section III-B for different values of the OFDM-symbol-normalized PDP tap drift rate $v_{\tau}=T \frac{v}{c}$, where $T$ is the OFDM symbol's duration, while $v$ and $c$ denote the speeds of the communicating terminal and light, respectively. Observe that the specific values of the parameter $v_{\tau}$ assumed in Figure 4 substantially exceed the maximum value considered in the base-line scenario outlined in Table I. Consequently, we may conclude that the CIR tracking method of Algorithm 1 exhibits an adequate performance over the entire range of practical channel conditions.

\section{PASTD AIDED DDCE}

The detailed schematic of the channel estimation scheme proposed is depicted in Figure 5. Our channel estimator is constituted by a bank of the per-subcarrier a posteriori MMSE CTF estimators [1], followed by the PASTD -aided CIR estimator of Algorithm 1 as well as by

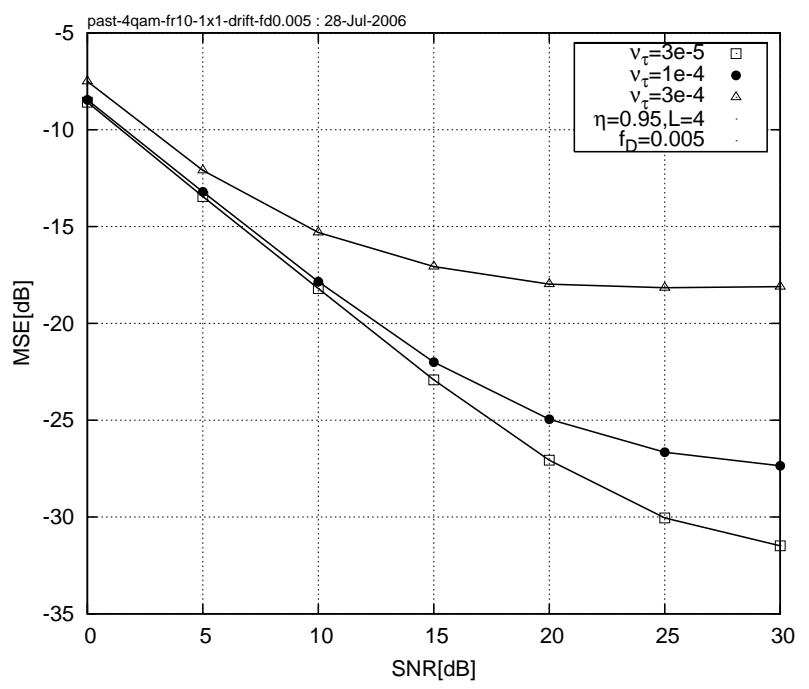

Fig. 4. MSE versus SNR performance exhibited by the 4QAM-OFDM system employing the PASTD method of Algorithm 1, while encountering different values of the PDP tap drift rate $v_{\tau}=3 \cdot 10^{-5}, 10^{-4}$ and $3 \cdot 10^{-4}$ as well as the Doppler frequency of $f_{D}=0.005$. Additional system parameters are summarized in Table I.

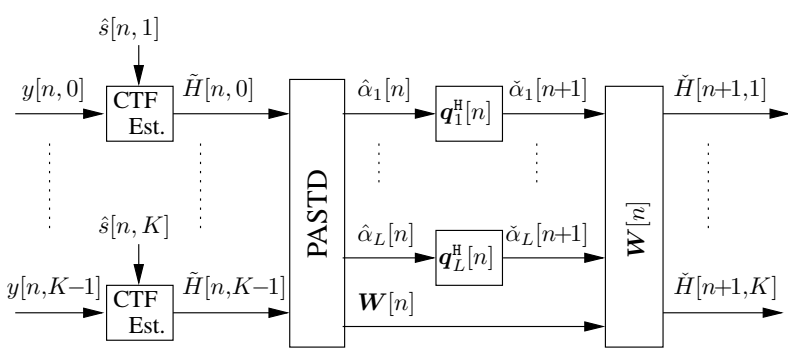

Fig. 5. Detailed structure of the $2 \mathrm{D}$ channel estimator corresponding to the DDCE module of Figure 1. The channel estimator comprises a PAST module, which performs recursive tracking of the CIR. The resultant CIR related taps $\hat{\alpha}_{l}[n]$ are filtered by the adaptive RLS-based prediction filter resulting in the a priori estimates of the CIR-related taps $\check{\alpha}_{l}[n+1]$. Finally, the a prior $i$ estimates of the subcarrier-related coefficients $H[n+1, k]$ are obtained by applying the transform matrix $W[n]$ provided by the PASTD module.

the a priori RLS CIR predictor [15]. The task of the CTF estimator seen in Figure 5 is to estimate the subcarrier-related CTF coefficients $H[n, k]$ of Equation (2). The resultant estimated subcarrier-related samples $\tilde{H}[n, k]$, which serve as an observation vector of the FD-CTF coefficients $H[n, k]$ are fed to the PASTD subspace-based tracking module, which performs recursive tracking of the channel's covariance matrix $C_{H}$ signal subspace and the associated CIR-related taps. The output of the PASTD module is constituted by the instantaneous

TABLE I

SYSTEM PARAMETERS.

\begin{tabular}{|l|c|}
\hline Parameter & Value \\
\hline \hline Carrier frequency $f_{c}$ & $2.5 \mathrm{GHz}$ \\
\hline Channel bandwidth $B$ & $8 \mathrm{MHz}$ \\
\hline Number of carriers $K$ & 128 \\
\hline FFT frame duration $T_{\mathcal{S}}$ & $16 \mu \mathrm{s}$ \\
\hline OFDM symbol duration $T$ & $20 \mu \mathrm{s}(4 \mu \mathrm{s}$ of cyclic prefix $)$ \\
\hline Max. delay spread $\tau_{\max }$ & $4 \mu \mathrm{s}$ \\
\hline Max. terminal speed $v$ & $130 \mathrm{~km} / \mathrm{h}$ \\
\hline Norm. Max. Doppler spread $f_{D}$ & $0.006=T \cdot 300 \mathrm{~Hz}$ \\
\hline Norm. Max. PDP tap drift $v_{\tau}$ & $2.4 \cdot 10^{-6} \mu \mathrm{s}=T \cdot 0.12 \mu \mathrm{s} / \mathrm{s}$ \\
\hline
\end{tabular}


CIR-related tap estimates $\hat{\alpha}_{l}[n]$ and the corresponding estimate of the transformation matrix $W[n]$ of Equation (16). The CIR-related estimate vector $\hat{\alpha}_{l}[n]$ is then fed into the low-rank time-domain CIRrelated tap predictor of Figure 5 for the sake of producing an a priori estimate $\check{\alpha}_{l}[n+1], l=1, \cdots, L$ of the next CIR-related tap-vector on a tap-by-tap basis [1]. Finally, the predicted CIR is converted to the subcarrier-related CTF with the aid of the transformation matrix $W[n]$ provided by the PASTD module of Figure 5 . The resultant FD-CTF is employed by the receiver for the sake of detecting and decoding of the next OFDM symbol. Note that this principle requires the transmission of a frequency-domain pilot-based channel sounding sequence, such as for example a pilot-assisted OFDM symbol, during the initialisation stage.

\section{A. Performance Results and Conclusions}

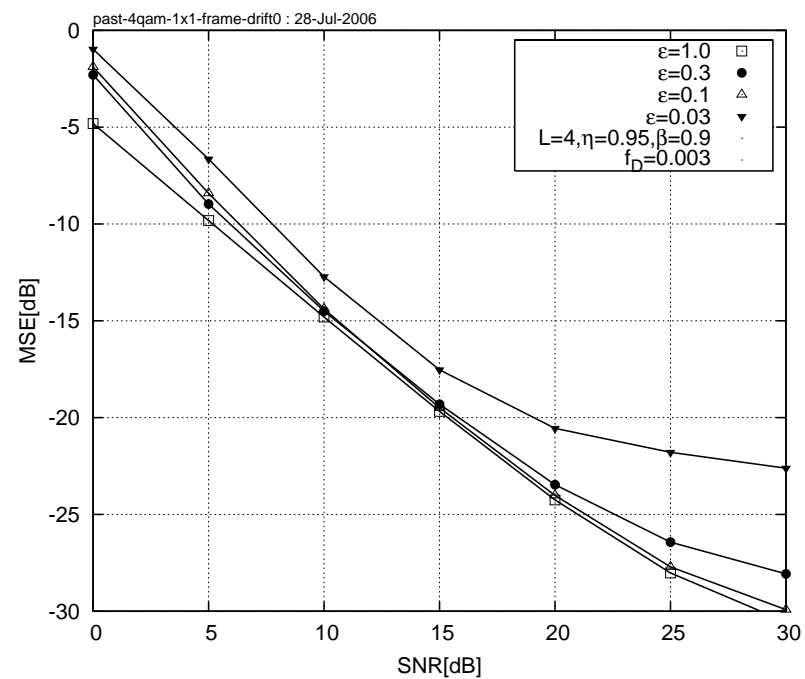

(a)

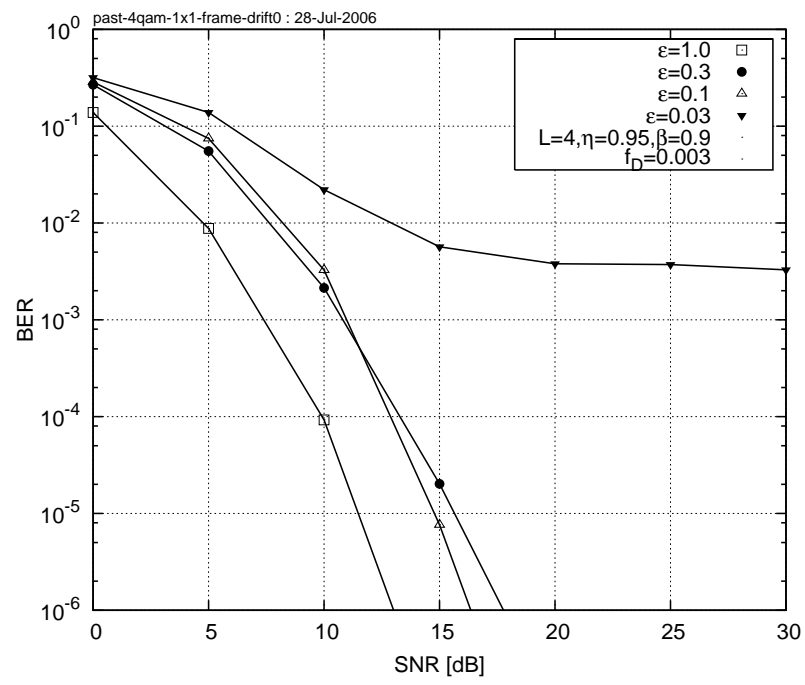

(b)

Fig. 6. (a) MSE and (b) BER versus SNR exhibited by the 4QAM-OFDM system employing the PASTD -aided DDCE. The value of the parameters $L=4, \eta=0.95$ and $\beta=0.9$ has been assumed. The Doppler frequency was $f_{D}=0.003$. Additional system parameters are summarized in Table I.

The achievable performance of the PASTD aided DDCE scheme proposed is characterized in Figure 6. In our simulations we considered an OFDM system having $K=128$ QPSK-modulated orthogonal subcarriers. The system characteristics are outlined in Table I. We employ an OFDM-frame-variant channel model associated with a time-variant 7-tap PDP characterized by the COST-207 BU channel model [14]. Additionally, each individual propagation path undergoes fast Rayleigh fading having an OFDM-symbol-normalized Doppler frequency of $f_{D}=0.003$. We assumed the values $L=4$ and $\eta=0.95$ for the PASTD module-related subspace rank and forgetting factor parameters respectively, as well as the value of $\beta=0.9$ for the RLS CIR-tap predictor-related forgetting factor. Additional system parameters are summarized in Table I.

Figure 6(a) portrays the achievable MSE performance of the PASTD aided DDCE for the pilot overhead ratios $\varepsilon=0.03,0.1,0.3$ and 1.0 , where $\varepsilon=0.03$ and $\varepsilon=1.0$ correspond to having $3 \%$ and $100 \%$ pilots, respectively. Furthermore, Figure $6(\mathrm{~b})$ portrays the corresponding BER performance of the rate $\frac{1}{2}$ turbo-coded QPSKmodulated OFDM system.

In conclusion, in this paper we have discribed a PAST-aided DDCE scheme. We have documented the achievable performance of the PAST-DDCE method proposed in the context of an OFDM system communicating in realistic channel conditions characterized by a time-variant fractionally-spaced PDP. We have demonstrated, that the channel estimation method proposed exhibits good performance over the entire range of relevant channel conditions, while using as little as $10 \%$ of pilot overhead.

\section{REFERENCES}

[1] L. Hanzo, M. Münster, B. J. Choi, and T. Keller, OFDM and MC-CDMA for Broadband Multi-User Communications, WLANs and Broadcasting. John Wiley and IEEE Press, 2003, 992 pages.

[2] A. Goldsmith, S. A. Jafar, N. Jindal, and S. Vishwanath, "Capacity limits of MIMO channels," IEEE Journal on Selected Areas in Communications, vol. 21, no. 5, pp. 684-702, June 2003.

[3] J.-J. van de Beek, O. Edfors, M. Sandell, S. Wilson, and P. Börjesson, "On channel estimation in OFDM systems," in Proceedings of Vehicular Technology Conference, vol. 2. Chicago, IL USA: IEEE, July 1995, pp. 815-819.

[4] O. Edfords, M. Sandell, J.-J. van de Beek, S. Wilson, and P. Börjesson, "OFDM channel estimation by singular value decomposition," IEEE Transactions on Communications, vol. 46, pp. 931-939, July 1998.

[5] Y. Li, L. Cimini, and N. Sollenberger, "Robust channel estimation for OFDM systems with rapid dispersive fading channels," IEEE Transactions on Communications, vol. 46, no. 7, pp. 902-915, April 1998.

[6] M.-X. Chang and Y. Su, "Model-based channel estimation for OFDM signals in Rayleigh fading," IEEE Transactions on Communications, vol. 50, pp. 540-544, April 2002.

[7] Y. Li, "Pilot-symbol-aided channel estimation for OFDM in wireless systems," IEEE Transactions on Vehicular Technology, vol. 49, pp. $1207-1215$, July 2000.

[8] B. Yang, "Projection approximation subspace tracking," IEEE Transactions on Signal Processing, vol. 43, no. 1, pp. 95-107, January 1995.

[9] Y. Li, "Simplified channel estimation for OFDM systems with multiple transmit antennas," IEEE Transactions on Wireless Communications, vol. 1, no. 1, pp. 67-75, January 2002.

[10] B. Yang, K. Letaief, R. Cheng, and Z. Cao, "Channel estimation for OFDM transmission in multipath fading channels based on parametric channel modeling," IEEE Transactions on Communications, vol. 49, pp. 467-479, March 2001

[11] S. M. Kay, Fundamentals of Statistical Signal Processing. Englewood Cliffs, NJ, USA: Prentice-Hall, 1998.

[12] S. Haykin, Adaptive Filter Theory. Englewood Cliffs, NJ, USA: Prentice-Hall, 1996.

[13] J. Yang and M. Kaveh, "Adaptive eigensubspace algorithm for direction or frequency estimation and tracking," IEEE Transactions on Acoustics, Speech and Signal Processing, vol. 36, pp. 241-251, 1988.

[14] M. Failli, "Digital land mobile radio communications COST 207," European Commission, Tech. Rep., 1989.

[15] D. Schafhuber and G. Matz, "MMSE and adaptive prediction of timevarying channels for OFDM systems," IEEE Transactions on Wireless Communications, vol. 4, no. 2, pp. 593-602, March 2005. 